\title{
Teaching System Identification. Goals and Formats for Different Courses
}

\author{
Lennart Ljung \\ Department of Electrical Engineering \\ Linkping University, S-581 83 Linkping, Sweden \\ WWW: http://www.control.isy.liu.se \\ Email: ljung@isy.liu.se \\ 2000-03-07

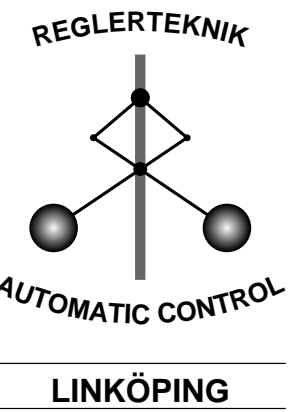

Report no.: LiTH-ISY-R-2222

For the IFAC Symposium on System Identification, SYSID2000,

Santa Barbara, CA, July 2000

Technical reports from the Automatic Control group in Linkping are available by anonymous ftp at the address ftp.control.isy.liu.se. This report is contained in the pdf file 2222.pdf. 


\title{
TEACHING SYSTEM IDENTIFICATION. GOALS AND FORMATS FOR DIFFERENT COURSES
}

\author{
Lennart Ljung* \\ * Division of Automatic Control, Linköping University, \\ SE-58183, Linköping, Sweden, email: ljung@isy.liu.se
}

\begin{abstract}
Four cases of System Identification courses are discussed and described: A short introduction to participants without any prior knowledge, a course for industry, an undergraduate course and a graduate course. The discussion is carried out in terms of how to convey six basic messages to the course participants.
\end{abstract}

\section{INTRODUCTION}

This contribution will deal with ideas and experiences of teaching system identification to four types of audiences:

(1) (Very) short courses for industrial audiences.

(2) Three-day courses for industrial audiences

(3) Undergraduate students

(4) Graduate students

Clearly, the approach to teaching and the mix of practical exercises and theoretical work will be quite different for these different situations. However, for any audience, and for any type of course, the following six basic questions and issues must be dealt with:

(1) How to adjust a family of models to measured data.

(2) How to visualize and evaluate the obtained models.

(3) How to understand the reliability of the estimated model.

(4) How to choose a model structure (a parameterized family of models).

(5) How to make sure that measured data are informative.

(6) How to perform the identification job in practice.

All these six basic questions must be illustrated and given partial answers for any of the audiences, even during a thirty minutes introduction to the topic. It is clear that the six questions can be penetrated to quite different depths and also illustrated both by theory and practical experiences at the computer. In the following sections I will describe my experience how to convey the six basic messages to a variety of course participants.

\section{A 30-MINUTE SYSTEM IDENTIFICATION COURSE}

What do you do if you are given 30 minutes to explain and illustrate system identification to an audience that may not know very much about signals and systems? Here is one possibility:

- Let the audience sit, possibly in teams of two, at computers using the System Identification Toolbox, (Ljung 2000). Use the toolbox only in the GUI mode.

- Instruct them to take a data set (like the hairdryer data), split it into two halves, and adjust a 4:th order state-space model to the first half. The character of the model is immaterial; just describe it as a family of possible descriptions being adjusted to observed data.

- Simulate the model using the other half of the data and compare measured output with model simulated output (i.e. push Model output). It's quite intuitive to explain what this plot tells us about the estimated model.

- Simulate a step response of the model, and show the confidence region around it. (push Transient response and turn on 
Confidence interval). Again, even if the audience is not familiar with the jargon on step responses, it is easy to explain what the plot shows, and the fact that the model has a notion of its own reliability.

- Let the audience try a couple of data sets where the signals and their relations have an intuitive appeal but may seem to be complex systems, like aileron deflections to pitch angles for an airplane etc

There are a few things to emphasize during such a short encounter with System Identification:

(1) Evaluate model behavior on validation data, not estimation data

(2) Models with surprisingly good reproduction capacity often can be achieved even with quite simple structures.

(3) Keep in mind, though, that this does not mean that they will behave equally well when tried out under other conditions.

\section{A COURSE FOR INDUSTRY}

The engineer's perspective on system identification can be crudely summarized by the following three questions:

(1) What shall I do with the data to come up with a model?

(2) How can I rely upon the model?

(3) What is the software support?

I have experience with trying to convey the answers to these blunt questions in anything from thirty minutes demonstrations of the system identification to a full week course on the topic.

The approach to dealing with the six basic questions, mentioned in the introduction can be as follows:

(1) Adjusting models to data The "curve fitting" interpretation will be quite sufficient in this context. It is easy to understand that if you parameterize a family of models by some vector of parameters you can evaluate how each model is able to reproduce the measured data. You than pick the model that shows the best reproduction performance based on the measured data. Linking this basic procedure to well known approaches like least squares is rather obvious, and it can also easily be understood that such schemes may have all kinds of statistical and probabilistic support once put in a stochastic framework. These aspects, however, are secondary for the industrial audience. It can be readily appreciated that a lot of work might be required to actually find the model that is closest to the measurement. From a pragmatic point of view, though, it is sufficiently reassuring to know that such algorithms exists in commercial software packages.

(2) Evaluating models. A problem with course participants that have not at all or not recently taken any systems course is that frequency domain interpretation, state space models, zeros and poles and the usual jargon might be unknown or forgotten. The most efficient visualization of the model properties is not doubt to look at the model's simulated output together with the measured output (that is the command compare in the system identification toolbox). This shows in very intuitive terms what the model is able to reproduce in the data and what it is not able to reproduce. Regardless of the formal understanding of linear systems etc these issues can be quickly understood by basically anyone. It is important to stress that at least this kind of evaluation of a model has to take place to give an indication of the confidence you may have for the model.

(3) Model reliability Even for an audience that has not really heard of step responses it is relatively easy to convey the notion of what the step response is. Then looking at such a response plotted with the confidence region corresponding to, say $99 \%$ is a good illustration of the variation in the estimate that can occur. This also gives a good opportunity to explain, in practical terms, the difference between just statistical uncertainty related to repeating the experiment, and the total model error. The compare plot shows the total model error including the additive noise, while the step response with uncertainty region just illustrates how much the step response may vary if the experiment would be repeated.

(4) Model structures The is the most tricky item. Now we have efficient code for multivariable state space models resulting from subspace methods in combination with prediction error methods for a free state-space parameterizations, (Ljung 2000), (Van Overschee and DeMoor 1996), (McKelvey and Helmersson 1999). Therefore, it is actually a good idea to first "sell" state space models. Even if the audience does not really know much about these representation the scalar measure of size (order) is an intuitive concept that is easy to cope with. If desired, all the model displays can be confined to compare, step etc., and one does not really have to understand the internal nature of the underlying model that generates these views.

The next step for model structure would be to talk about input delays. Again, the notion of delays from the input is easy to 
understand, even without detailed structure analysis. The message is then that a rather versatile model structure is obtained by trying to find out what the delays are and then combine them with model "size", just one scalar number.

In a longer course for industrial audiences, more time is spent on model structures, see below. The simple ARX model as "the mother of all models" is agood starting point, just explaining in a simple way how the next output can be computed or "predicted" from past data for particular parameter values:

$$
\begin{aligned}
y(t)= & -a_{1} y(t-T)-\ldots-a_{n} y(t-n T) \\
& +b_{1} u(t-T)+\ldots+b_{m} u(t-m T)
\end{aligned}
$$

(5) Experiment design. In a short course for industry it is usually not possible to deal very much with experiment design. What I usually do is to illustrate that a step input is not sufficient to estimate more than a very simple model, and I also explain the reasons for that. That is sufficient to put the finger on the fact that the input has to be exciting.

(6) How to do it. In this context I recommend a simple rule of thumb procedure to follow:

(a) Estimate a default order state space model using a prediction error method in a free parameterization:

model = PEM (data)

Compare the model output of this model with the measured output for a validation part of the data.

compare (model, datav)

Compare the separate responses of this model with those of non-parametric method:

step (model, data)

(b) Judge if the compare command gives a reasonable reproduction of the measured signal. If the basic features of the data behavior are present in the model output, do a little bit of fine tuning of model order and delays. If the step responses show a clear delay of $n$ samples, enforce that delay into the state space model: model = PEM (data, $\mathrm{nx}, 3$, 'nk', [3 4 0]

In the multivariable case, check from the step response estimates if some inputs have negligible influence on the outputs. If so, try a model without that input and see if its capability of reproducing the output is significantly changed or deteriorated. Also, try a few model orders around the automatically chosen one to see if there are any significant changes in the model behavior.

If the compare plot is not satisfactory, then think over if more inputs should be added to be better able to explain or reproduce the output. Also, do "semi physical modeling", that is, consider what is known about the physics of the system. Introduce non-linear transformations of the measured data, that seem to be reasonable for the physical configurations (like squaring voltage to create something that is proportional to power) and introduce the various non-linear transformations as extra inputs to the model. If that does not help, try non-linear black box structures like neural networks and the like.

One of the most important messages to get through to this audience is to split the data into estimation data and validation data, and to explain why this is necessary. Also to convey an understanding of why a model can be deficient: unsuitable structure, and/or noisy measurements.

There may be a tendency from an academic point of view to over-emphasize the detailed choice of model orders like in ARMAX models etc. I think it is important to stress that it is more relevant to focus on which inputs to include in model, and to be keen on detecting non-linear effects. For practical data this is usually much more important than fine tuning the different structures that may be involved.

For industrial courses that last more than one day, most of the extra time is spent on a deeper discussion of model structures: How to incorporate (semi) physical knowledge. Input-output models of ARMAX output error type and how non-linear black box models such as neural networks fit into this general framework. I also, in those cases go a little bit deeper in experiment design, talk about excitation and input spectra, and also of advantages of periodic inputs etc.

\section{AN UNDERGRADUATE COURSE}

At Linköping University we have been teaching a course with the title "Modeling and Simulation" for the past fifteen years. It contains about $40 \%$ physical modeling, including bond graphs, some $40 \%$ on system identification, and about $20 \%$ regarding simulation techniques and software for simulation of dynamical systems. The course is given to the fourth year undergraduates and is taken by about 100 students every year. The textbook is (Ljung and Glad 1994) and the course outline pretty much follows that text.

The whole course has 12 2-hour lectures, 12 2hour problem solving, and computer sessions and 12 hours of laboratory work. 
In terms of the six basic questions the goal is to convey the following perspectives:

(1) Adjusting models to data We stress the common basis and simple philosophy of a prediction error method: A model is a predictor, and parameter estimation methods are optimization techniques to make these predictors perform as well as possible on measured data. The links to the basic statistical techniques such as maximum likelihood and least squares, that the students have seen in their undergraduate statistics courses are fully pointed out. A derivation of the maximum likelihood for dynamical systems is included, showing that this becomes a "prediction error method".

(2) Evaluating models. The undergraduate students have recently taken courses in signals and systems, and automatic control courses which means that it is easy to point to various means of evaluating the models properties: zeros and poles, frequency functions, etc. It is also easy to emphasize that these things should be looked at including the confidence regions or uncertainty regions that come from the parameter uncertainty.

(3) Model reliability. A simple derivation, without all the technical details of the convergence properties of parameter estimates, as well as their asymptotic distribution is included in the course. The kinship with similar well known results for linear regressions and the linear least square method is pointed out. While the variance aspect of the model error is very much parallel to the treatment the students have seen in statistics, the notion of bias (actually undermodeling, which is different from "bias" in statistics) may require some more work.

The characterization

$$
\begin{aligned}
\hat{\theta} & =\arg \min _{\theta} V(\theta) \\
V(\theta) & =\int\left|G_{0}\left(e^{i \omega}\right)-G\left(e^{i \omega}, \theta\right)\right|^{2} \frac{\Phi_{v}(\omega)}{\left|H_{*}\left(e^{i \omega}\right)\right|^{2}} d \omega
\end{aligned}
$$

for the limiting linear model $G(q, \hat{\theta})$ if the true system $G_{0}$ is linear is quite useful and intuitive. It helps pinpointing the character of the model approximations involved, and the nature of the bias.

(4) Model structures In this course we treat all the typical input-output model structures like Box-Jenkins, output error, ARMAX and ARX, as well as general parameterized linear and non-linear state space models. Recently we have also included material that shows how artificial neural networks relate to the general framework.

(5) Experiment design. A fairly pragmatic treatment of frequency contents of the input sig- nal, problems with feedback, pre-filtering of data, removal of outliers and trends is included.

(6) How to do it? In addition to the simple scheme described in the previous section a discussion about the choices among different input-output models like ARX, ARMAX and Box-Jenkins is included, as well as the Akaike criterion, AIC, the final prediction error criterion, FPE, and Rissanen's minimal description lengths, MDL, are also described. The basic techniques of residual analysis as model validation are described and the confidence regions for the correlation estimates are derived.

The education focus is to mix the above material with hands-on experience at numerous computer sessions dealing with real data, as well as laboratory sessions where data are collected from a laboratory process.

\section{A GRADUATE COURSE ON SYSTEM IDENTIFICATION}

The graduate, Ph.D., course on system identification given in Linköping is intended for students who already have taken the undergraduate course described in the previous section. The textbook used is (Ljung 1999). The sub-title "Theory for the User", emphasizes the focus of also the graduate course. The goals are set as follows:

- Understand the mathematical/probabilistic framework. Quasistationary signals, ergodicity, almost sure (uniform) convergence, convergence in distribution. Several students have a quite rudimentary background in stochastic theory.

- Be able to derive quality measures, in terms of bias, variance and confidence intervals, for estimated models.

- Understand the interplay between the formal mathematical setup and quality measures of practical interest.

- Be able to design good inputs/experiments based on (simple) prior knowledge and intended model use.

- Have experience to build models from real, industrial data. Understand model structure selection, and how to deal with feedback from model validation results.

The graduate course rather much follows the chapters of the textbook. It is typically laid out over 8 weeks, with one 2-hour lecture per week, dealing with 2 chapters at a time. The lectures are a mixture of discussions of the previous week's material and introducing the next week's material. In addition, the students meet regularly to 
discuss the assigned problems (taken from the book). Moreover the students form teams to deal with real data sets from different aspects.

The web page

http://www. control. isy. liu.se/ ljung/idkurs/idkurs.html

contains more of the logistic details.

In terms of the 6 basic questions, the ambitions of the course are as follows:

(1) Adjusting models to data The full spectrum of interpretations of parametric methods, in terms of "curve-fitting" (minimizing prediction errors), maximum likelihood, with its various aspects, regularization, numeric search, gradient calculation etc. is covered, as well as the frequency domain interpretations/variants. Fourier analysis in terms of the ETFE, and spectral analysis as a smoothing of the ETFE is also included. Subspace methods are treated first pragmatically as state-estimation from predictions followed by a least-squares step for known states, and then with all the numerical details.

(2) Evaluating models. A rather thorough treatment of residual analysis in included, in addition to all the features dealt with in the undergraduate course.

(3) Model reliability. Perhaps the most demanding part of the course is to penetrate the asymptotic analysis. Understanding how to derive limiting models, and their asymptotic distributing is important, as well as translating the basic parametric bias/variance notions to quantities of greater practical importance. The "asymptotic, asymptotic" quality measures of the frequency function, their use and limitations, play an important role.

(4) Model structures The course covers a general predictor model setup, including the standard linear input-output and state space models, and arbitrarily parameterized linear models in discrete and continuous time. It also covers non-linear models with a special emphasis of the general non-linear black-box model family, that includes artificial neural networks, wavenets and fuzzy models.

(5) Experiment design. The notions of informative experiments, optimizing data information contents, as well as typical and useful inputs are treated in some detail. PRBS, sum of sinusoids as well as random inputs are discussed, as well as aspects on periodic inputs and the intersample behavior of the input. The problems and possibilities with output feedback is another important topic.

(6) How to do it. The students form teams to deal with a number of industrial data sets parallel to studying the book and solving paper-and-pencil problems. The DAISY website

http://www.esat.kuleuven.ac.be/sista/daisy/ is an excellent source for finding interesting and challenging data sets.

\section{REFERENCES}

Ljung, L. (1999). System Identification - Theory for the User. 2nd ed.. Prentice-Hall. Upper Saddle River, N.J.

Ljung, L. (2000). System Identification Toolbox for use with MatlaB. Version 5.. 5th ed.. The MathWorks, Inc. Natick, MA.

Ljung, L. and T. Glad (1994). Modeling of Dynamic Systems. Prentice Hall. Englewood Cliffs.

McKelvey, T. and A. Helmersson (1999). A dynamical minimal parametrization of multivariable linear systems and its application to optimization and system identification. In: 14th World Congress of IFAC. Vol. H. IFAC. Beijing, China. pp. 7-12.

Van Overschee, P. and B. DeMoor (1996). Subspace Identification of Linear Systems: Theory, Implementation, Applications. Kluwer Academic Publishers. 